\title{
El camino de la Unión Europea hacia una estrategia de relaciones culturales internacionales
}

\author{
Edith Ruvalcaba Galindo ${ }^{1}$
}

\begin{abstract}
Resumen
La Unión Europea (UE) ha desarrollado una política exterior sólida, integrada por políticas comunitarias e intergubernamentales. Si bien la cultura ha estado presente desde los comienzos del proceso de integración, ésta se ha percibido mediante los esfuerzos individuales en el nivel nacional. No obstante, la creciente demanda por una política comunitaria y el avanzado nivel de integración exigen como siguiente paso una propuesta para una estrategia común de relaciones culturales internacionales. El documento tiene por objetivo describir el camino que ha seguido la UE en la materia; aborda la elección del término relaciones culturales internacionales sobre el de diplomacia cultural; y analiza los esfuerzos previos, encontrando que la política cultural de la Unión Europea se basa en tres líneas de acción: promoción del diálogo intercultural para el establecimiento de relaciones pacíficas, apoyo a la cultura como motor del desarrollo económico y social y la cooperación en materia de patrimonio cultural.

Palabras clave: Unión Europea, política cultural, relaciones culturales, diplomacia cultural, política exterior
\end{abstract}

1. Centro Universitario de Ciencias Económico Administrativas (CUCEA). Maestría en Relaciones Económicas Internacionales y Cooperación (con énfasis en la Unión Europea y América Latina). Universidad de Guadalajara, México. Correo electrónico: edithruvalcaba@ gmail.com 


\title{
THE PATH OF THE EUROPEAN UNION TOWARDS A STRATEGY
}

OF INTERNATIONAL CULTURAL RELATIONS

\begin{abstract}
The European Union has developed a solid foreign policy, with common policies as well as intergovernmental agreements. In spite of the cultural dimension being present since the beginning of the integration process, cultural policies remained at the national level of its member States. However, the ongoing demand for a common policy, as well as the advanced level of integration, calls for a natural step: an EU strategy for international cultural relations. With the aim to describe the path followed by the Union in the cultural field, the document engages on the EU's decision to use the term "international cultural relations" over others, such as cultural diplomacy; it approaches the previous actions, finding that the EU cultural policy it is based on three main principles: the promotion of intercultural dialogue towards the establishment of peaceful relations, support for culture as the engine of economic growth and social development, as well as cooperation for the cultural heritage protection.

Keywords: European Union, Cultural Policy, Cultural Relations, Cultural Diplomacy, Foreign Policy
\end{abstract}

\section{Introducción}

Bajo el lema "Unida en la Diversidad", la Unión Europea ha logrado consolidarse como el proceso de integración más avanzado en las diferentes dimensiones: político, económico y social. En su integración, ha desarrollado una política exterior sólida y prácticamente única en el mundo, en cuanto a su naturaleza supranacional. No obstante, existe otro pilar fundamental, que si bien ha estado presente desde sus inicios, hasta hace unos años no había sido integrado formalmente dentro de las políticas comunitarias: la cultura. Bajo el marco del Tratado de Funcionamiento de la UE, la política cultural se alberga en el ámbito nacional, respetando la soberanía de cada uno de los Estados miembros.

Si bien las diferencias culturales han fortalecido el proceso, también han implicado la búsqueda de una identidad europea y, por ende, la necesidad de una política común en la materia. Aunado a lo anterior, diversos sucesos que han tenido lugar en el escenario internacional han posicionado a la cultura como un pilar fundamental en las relaciones internacionales de los últimos años. 
Mediante la evidencia de distintas acciones y esfuerzos por parte de la Unión y sus instituciones en la esfera cultural, especialmente en materia de la cooperación cultural, y ante la reciente Comunicación Conjunta de la Comisión y el Parlamento Europeo para una Estrategia de la UE para las Relaciones Culturales Internacionales; parece que finalmente nos encontramos ante la materialización de una política cultural común.

El presente documento tiene por objetivo trazar y describir las acciones que ha seguido la Unión Europea en materia de cooperación cultural hasta llegar a una estrategia de relaciones culturales internacionales.

El trabajo se estructura en cuatro secciones. Comienza con un breve marco teórico que permite comprender la elección de la UE por el termino relaciones culturales internacionales sobre otros más comunes en la esfera internacional, como diplomacia cultural. En un segundo momento se incluyen los antecedentes y los primeros esfuerzos de cooperación cultural en el continente, no necesariamente dentro del marco de la Unión.

Una tercera sección aborda específicamente el camino de la Unión Europea en materia cultural, se incluyen algunos esfuerzos importantes como la Agenda Europea para la Cultura, el Informe sobre las Dimensiones Culturales de las Acciones Exteriores de la UE presentado por la eurodiputada Marietje Schaake en 2011, la Acción Preparatoria sobre "La Cultura en las Relaciones Exteriores de la UE", entre otros; para concluir con el documento que titula a la presente investigación, "Hacia una estrategia de la UE para las relaciones culturales internacionales", publicado en junio de 2016. Finalmente se incluyen algunas conclusiones sobre las coincidencias en las distintas acciones de la Unión, lo que permite detectar los ejes centrales de la dimensión cultural en la actuación de la Unión como agente global.

\section{Marco teórico}

Tras varios años en donde la esfera global centraba su atención en el equilibrio de poder militar y económico, la dimensión cultural ha ido adquiriendo mucha mayor importancia. Si bien los factores culturales han estado presentes en las relaciones internacionales desde varios 
siglos atrás, no es hasta finales del siglo XX y principios del siglo XXI que la cultura se posiciona como un pilar fundamental en la política externa de los Estados, junto al comercio (economía) y la política (seguridad) (Saddiki, 2009).

Son varios los elementos que han contribuido a la emergencia y posicionamiento de la cultura en la política internacional. Por una parte, las interacciones entre distintas sociedades, cada vez más frecuentes gracias al proceso globalizador y los avances tecnológicos; las recientes guerras movilizadas a través de los tintes religiosos y diferencias étnicas (Pantea \& Stoica, 2009; Saddiki, 2009); o bien el importante papel que desempeña la cultura en el crecimiento económico y el progreso, y su habilidad para influir entonces en la disminución de las grandes brechas sociales y económicas (PNUD, 2005). Por último, algunos de los retos que enfrenta la humanidad del siglo XXI -como el cambio climático, terrorismo y la migración - no tienen solución únicamente en el poder militar y/o económico, es necesario incluir la dimensión cultural para abordarlos (Bound et al., 2007).

Especialmente en Europa, prestar atención al aspecto cultural se vuelve importante por factores como el aumento de la intolerancia, discriminación y xenofobia en una sociedad que promueve el respeto y la tolerancia ante las diferencias, lo que podría resultar en problemas de seguridad (Consejo de Europa, 2011). Aunado a esto, la diversidad cultural ha sido una parte fundamental en los valores de la Unión Europea, indicado así en su propio lema "Unida en la Diversidad".

Lo anterior ha dado pie al surgimiento de nuevas áreas de estudio en la academia. Uno de los conceptos más utilizados actualmente en el campo internacional es el de diplomacia cultural. Se distingue por la confusión y debate que gira en torno a su definición. Entre las posturas de los distintos académicos, diplomáticos y líderes de opinión, se observan diversos puntos de vista en cuanto a sus objetivos, herramientas y actores. No obstante, una de las definiciones más citadas en el campo es la de Milton Cummings, que la detalla como:

[...] the exchange of ideas, information, art, and other aspects of culture among nations and their peoples in order to foster mutual understanding. But 'cultural diplomacy' can also be more of a one-way street than a two-way exchange, as when one nation concentrates its efforts on promoting the national language, explaining its policies and 
point of view, or 'telling its story' to the rest of the world ${ }^{2}$ (Cummings, 2003, pág. 1).

Otros conceptos de relevancia son el de diplomacia pública ${ }^{3}$ y poder suave. El primero hace referencia al uso de las dimensiones culturales por parte de un gobierno, en un discurso dirigido hacia un público de otro país, con la finalidad de alcanzar intereses nacionales. Mientras que el segundo es utilizado por el estadounidense Joseph Nye para describir "la habilidad de lograr lo que queremos mediante la atracción, en vez de recurrir a la coerción o a sanciones económicas. Surge de la capacidad de atracción de la cultura de un país, de sus ideales políticos y de sus políticas" (Nye, 2016, pág. 20).

Empero, la UE ha optado por utilizar su propio término, relaciones culturales internacionales (Isar, 2013). Acorde a uno de los líderes del consorcio responsable de la Acción Preparatoria sobre la "Cultura en las Relaciones Exteriores de la UE", dicha decisión le permite a la Unión un margen de acción más amplio, así como la previene de caer en las "trampas" que conlleva el término de diplomacia cultural. En su documento "Culture in External Relations: The EU in Cultural Diplomacy", Isar lo califica de ambiguo y confuso, discutiendo que el concepto se ha alejado de la especificidad de significado, abrazando en la actualidad, prácticamente cualquier actividad relacionada con la cooperación cultural, además de definir objetivos utópicos y fuera de su alcance.

Para complementar su argumento, Isar sostiene que la diplomacia cultural ha fallado en la agrupación de los esfuerzos de los distintos actores del sistema internacional (estatales y no-estatales), ${ }^{4}$ en tanto que aquellas relaciones culturales "informales" son mucho más efec-

2. Traducción personal: "el intercambio de ideas, información, arte y otros aspectos de la cultura, entre las naciones y sus pueblos para fomentar el mutuo entendimiento. Pero la 'diplomacia cultural' puede ser también más una calle de un solo sentido que un intercambio mutuo, es decir, cuando una nación concentra sus esfuerzos en promover el propio idioma, explicar sus políticas y su punto de vista o 'contar su historia' al resto del mundo."

3. Utilizado por primera vez en 1965 por Edmund Guillion, maestro de la Universidad de Tufts, en EE.UU. para sustituir el término de "propaganda".

4. La diplomacia cultural surge como disciplina en la década de los 1990s (aprox.), tras el surgimiento de nuevos actores en el sistema internacional, con el fin de la Guerra Fría; así como los avances tecnológicos que les permitieron prácticamente el mismo alcance que anteriormente era exclusivo del Estado. Dicho escenario, ha complicado el debate sobre quiénes son los responsables de practicar la disciplina, el Estado y/o además los actores no-estatales. 
tivas que los esfuerzos de los organismos estatales. Por otra parte, argumenta que hasta la fecha no existe ningún documento que sustente que efectivamente la práctica formal de la disciplina ayude a disminuir la imagen negativa de una nación. Por último sostiene que las diferencias culturales no son causa de conflicto por sí solas, sino la politización de las diferencias, la que motiva y exacerba los problemas.

Teniendo en cuenta lo anterior, el término cultura en las relaciones exteriores, mucho más general y extenso en su contenido, permite a la Unión Europea abarcar las distintas dimensiones culturales dentro del campo de las relaciones internacionales, así como sus herramientas, actores, enfoques y objetivos en su política exterior.

\section{Antecedentes}

La palabra "Europa" tiene sus raíces etimológicas en los prefijos griegos eu, que significa verdadero y opsis, que expresa vista u ojos; y juntos se entienden como "ojos grandes" (Roulet, 2017). Por otra parte, en la tradición del mundo clásico, Europa era una mujer de mirada bonita y ojos grandes (Martín de la Guardia; Pérez, 2001). Entonces, es posible inferir que Europa posee una visión grande, hacia el futuro.

Lo que en un principio constituía una utopía, logró constituirse tras la devastación de dos guerras mundiales. Con la firme decisión de poner fin al odio y rivalidad entre los países europeos y motivados por grandes líderes como Robert Schuman, Jean Monnet, Alcide de Gasperi, Konrad Adenauer y Winston Churchill, éstos se unieron en su deseo de encontrar la paz mediante la integración (Fontaine, 2014).

Con una "mirada hacia lo grande", Europa ya no puede definirse sólo en términos geográficos. Se trata más bien de un concepto cultural e histórico (Gorzegno, 2017). Lo que inició con un proceso de integración económica y política, ha logrado evolucionar y crecer, viendo más allá de sus diferencias y encontrándose en puntos de interés común para fortalecerse en su diversidad. De hecho, podría decirse que dentro del estudio de los procesos de integración regional, la Unión Europea es el modelo más avanzado y único en su género, logrando extenderse al aspecto social, bajo la búsqueda de una identidad europea, tanto al interior, así como en la promoción de los valores europeos al exterior. 
En su integración, la UE y sus instituciones, han desarrollado una política exterior sólida, que actúa en una serie de ámbitos de importancia para sus Estados miembros (Fontaine, 2014). Se compone de tres pilares fundamentales: económico (comercial), político (seguridad) y de cooperación.

La política exterior de la UE se integra por políticas comunitarias e intergubernamentales. En las primeras se encuentran las relaciones exteriores de la Unión, específicamente la política comercial (económica) y de cooperación. Mientras que el aspecto intergubernamental abarca el diálogo político y de seguridad que, como su nombre lo indica, corresponde a las políticas nacionales de los Estados miembros.

Al día de hoy las políticas comunitarias incluían únicamente los aspectos económicos y de cooperación. No obstante, la UE - en su carácter de proceso de integración regional avanzado - no ha integrado en sus políticas uno de los pilares fundamentales de las relaciones internacionales: la cultura. La Unión respeta la soberanía de sus Estados miembros, dejando la responsabilidad de la política cultural en el nivel nacional de cada uno de ellos, siendo papel de la Comisión Europea únicamente el de ayudar a hacer frente a los retos comunes en materia cultural.

Al respecto, el artículo 167, Título XIII del Tratado de Funcionamiento de la Unión Europea, en su versión consolidada, menciona lo siguiente:

2. La acción de la Unión favorecerá la cooperación entre Estados miembros y, si fuere necesario, apoyará y completará la acción de éstos en los siguientes ámbitos:

- la mejora del conocimiento y la difusión de la cultura y la historia de los pueblos europeos,

- la conservación y protección del patrimonio cultural de importancia europea,

- los intercambios culturales no comerciales,

- la creación artística y literaria, incluido el sector audiovisual.

3. La Unión y los Estados miembros fomentarán la cooperación con los terceros países y con las organizaciones internacionales competentes en el ámbito de la cultura, especialmente con el Consejo de Europa.

Como el Tratado establece, pese a la falta de una política cultural comunitaria, existen varios esfuerzos en materia cultural, sobre todo en el ámbito de la cooperación. Éstos se encuentran en otros niveles, me- 
diante proyectos de colaboración de las instituciones de la Unión Europea, relaciones bilaterales de los Estados miembro, o bien, mediante el esfuerzo de instituciones de otra naturaleza, como el Consejo de Europa, ${ }^{5}$ o EUNIC. ${ }^{6}$ Cabe mencionar además que, los Estados europeos son pioneros en el establecimiento de institutos de promoción cultural (Paschalidis, 2009), sobre todo Francia, nación que se ha distinguido por hacer de su cultura su carta de presentación ante el mundo, bajo la frase 'la culture c'est la politique'.

En cuanto al Consejo de Europa, sus actividades en el ámbito cultural se fundamentan en 4 principios: respeto a la diversidad cultural, promoción de la identidad, participación y acceso a la cultura, así como el apoyo a la creatividad (Consejo de Europa "Compendium of Cultural Policies and Trends in Europe", 2016). Actualmente el campo de la cultura en el marco institucional del Consejo de Europa compete al Comité Director de Cultura, Patrimonio y Paisaje (CDCPP) creado en 2012.

Bajo el Convenio Cultural Europeo, firmado en París en 1954, los Estados europeos y miembros del Consejo de Europa firmaron el Tratado, comprometiéndose al desarrollo de la cooperación cultural para el entendimiento entre las personas de Europa, así como la apreciación de la diversidad cultural y el objetivo de una política de acción común (Consejo de Europa, 2017), mucho antes que cualquier iniciativa de la Unión Europea en la materia.

Si bien tanto el Consejo de Europa como la Unión Europea encuentran sus antecedentes en los ideales de la integración posterior a la II Guerra Mundial. La primera se consolida con el Tratado de Londres (1949) y adquiere un objetivo solido de protección de los derechos hu-

5. El Consejo de Europa es una organización internacional, ajena a la Unión Europea, y de carácter regional, cuyo principal objetivo es la defensa y protección de los derechos humanos, la democracia y el Estado de Derecho, mediante la velación de la implementación y desarrollo del Convenio Europeo para la Protección de los Derechos Humanos y de las Libertades, firmado el 4 de noviembre de 1950. El Consejo se integra de 47 Estados miembros, incluyendo a los 28 miembros de la UE, prácticamente todos las naciones europeas, a excepción de Bielorrusia y Kazajistán.

6. El European Union National Institutes for Culture (EUNIC) es una red que aloja a los institutos culturales de los 28 Estados miembros de la UE, comprometidos con la acción cultural más allá de sus fronteras. Su objetivo es unir los distintos esfuerzos nacionales hacia un proyecto comunitario europeo, así como la promoción de la diversidad cultural y el diálogo intercultural y abogar por el papel de la cultura en las políticas externas, tanto a nivel europeo como internacional. 
manos tras la firma del Convenio Europeo de Derechos Humanos en Roma (1950). Por su parte, los primeros antecedentes históricos de la Unión Europea se remontan a la creación de la Comunidad Europea del Carbón y del Acero (CECA) en 1951, unión de carácter económico y posteriormente político. La naturaleza de ambas instituciones en sus inicios permite comprender por qué la experiencia en el ámbito cultural del Consejo de Europa es tanto más antiguo como extenso que el de la Unión Europea.

No obstante, ambas organizaciones han colaborado en varios programas del sector cultural. En 2006, en colaboración con la Comisión Europea y el Foro Europeo de la Juventud, el Consejo de Europa lanzó la campaña "All Different-All Equal Campaign for Diversity, Human Rights and Participation", ${ }^{7}$ con la finalidad de promover el respeto ante la igualdad universal y las diferencias específicas.

Otros proyectos realizados en conjunto, incluyen el "European Heritage Day" (Jornadas Europeas de Patrimonio), creado en 1991 con el objetivo de promover el acceso al patrimonio cultural de Europa. Y desde 2008, el programa RECI, Red de Ciudades Interculturales, con la finalidad de apoyar a las ciudades en la revisión de sus políticas para el desarrollo de estrategias interculturales que les permitan afrontar positivamente la diversidad cultural (Consejo de Europa, 2017).

En cuanto a las acciones propias de la Unión Europea, un momento clave es su participación en la Convención sobre la Protección y Promoción de la Diversidad de las Expresiones Culturales, de la UNESCO. En 2005, todos los Estados miembros de la UE de manera individual, así como ésta en su conjunto, firmaron la Convención, comprometiéndose a "proteger y promover la diversidad de las expresiones culturales; crear las condiciones para que las culturas puedan prosperar y mantener interacciones libremente [...]; fomentar el diálogo entre culturas [...]; fomentar la interculturalidad [...]; reafirmar la importancia del vínculo existente entre la cultura y el desarrollo [...]" (UNESCO, 2005, pág. 4), entre otros. Lo anterior representó para la UE la importancia de fortalecer al sector cultural así como, a través de éste, estrechar lazos más firmes con otras regiones en el mundo (Unión Europea, 2014).

7. Traducción personal: “Todos Diferentes-Todos Iguales, campaña para la Diversidad, Derechos Humanos y Participación”. 
Por otra parte, en 2006 la Fundación Europea de la Cultura ${ }^{8}$ publicó algunos estudios sobre el papel de la cultura en las relaciones exteriores de la UE, concluyendo que no había razón o impedimento alguno que le permitiera a la Unión avanzar hacia una política cultural comunitaria. Los estudios además establecieron los objetivos de la acción cultural en tres sectores: seguridad, visibilidad y desarrollo económico, a llevarse a cabo a través del establecimiento del diálogo intercultural y la promoción de los valores europeos en el exterior (Unión Europea, 2014).

El mismo año surge EUNIC, la red que agrupa a los institutos culturales europeos, pero ajena a la UE. Ésta tiene como visión “By 2025, EUNIC will be the delivery, research and training partner of choice for cultural diplomacy and cultural relations at European and international level." ${ }^{\prime 9}$ Cabe resaltar su referencia a ambos conceptos, "diplomacia cultural" y "relaciones culturales", conceptos que definirían la acción cultural exterior de la Unión Europea más adelante.

Hasta el día de hoy, los esfuerzos de la Unión en políticas comunitarias se centraban específicamente en los ámbitos comercial y de cooperación, pero con poco énfasis en la cooperación cultural. Prácticamente ésta y todo lo relacionado al sector cultural correspondía exclusivamente a la soberanía de sus Estados miembros. No obstante, lo anterior estableció el camino que seguiría la Unión hasta llegar en junio de 2016 a acciones más concretas con la Comunicación Conjunta al Parlamento Europeo y al Consejo "Hacia una estrategia de la UE para las relaciones culturales internacionales".

8. La Fundación Europea de la Cultura (European Cultural Foundation [EFC]), es una organización independiente a la Unión Europea, establecida en 1954 en Ginebra (actualmente tiene sus instalaciones en la ciudad de Ámsterdam) bajo la visión de reconstruir a la Europa de la post-guerra a través de la cultura. En su trabajo se encuentra el apoyo y establecimiento de redes entre los distintos agentes del sector cultural.

9. Traducción personal: "Para el 2025, EUNIC será el socio responsable de investigación y formación para la diplomacia cultural y las relaciones culturales, tanto a nivel europeo como internacional." 


\section{La Unión Europea y el establecimiento de una estrategia para las relaciones culturales internacionales}

\section{Agenda Europea para la Cultura en un Mundo en vías de Globalización}

En mayo de 2007, la Comisión Europea presentó al Parlamento, al Consejo, al Comité Económico y Social Europeo y al Comité de las Regiones, la Comunicación sobre una Agenda Europea para la Cultura en un Mundo en vías de Globalización. En ésta se establecía el papel central de la cultura como núcleo para el desarrollo humano, y comprendía la importancia que ha jugado en el proceso y éxito de su integración.

Dicho documento establecía la importancia de fortalecer al sector cultural ante los nuevos retos del escenario internacional; ante la importancia del diálogo intercultural para establecer un ambiente de paz; debido a su aportación al papel influyente de Europa en el mundo y su contribución a la promoción de una sociedad incluyente; y por último, su importancia en el sector económico mediante la creación de empleos y el comercio de bienes y servicios culturales.

Tras una revisión de las acciones que hasta entonces había llevado a cabo la UE en materia cultural, así como otras más programadas a llevarse a cabo durante los siguientes años, la Comisión concluía la importancia de desarrollar una agenda cultural común, que permitiera a la Unión establecer nuevos métodos de cooperación entre los Estados miembros, la sociedad civil y terceros países (Comisión de las Comunidades Europeas, 2007).

La Comunicación determinaba tres categorías de objetivos a establecerse en la Agenda común en materia cultural:

- Promoción de la diversidad cultural y el diálogo intercultural: Incluyendo acciones para promover el diálogo y las competencias interculturales, como la movilización de artistas y recursos y la circulación de expresiones artísticas.

- Promoción de la cultura como catalizador para la creatividad en el marco de la Estrategia de Lisboa para el Crecimiento y el Empleo: Ante la importancia que representa el sector cultural en la economía, ${ }^{10}$ se

10. En 2004, el 3,1\% de la población empleada total de la UE (entonces de 25 Estados miembros), trabajó en el sector cultural. Además, éste contribuyó al PIB de la UE en 2,6\% en 2003 (Comisión de las Comunidades Europeas, 2007, pág. 10). 
pretendía promover el desarrollo de habilidades de gestión y administración para el sector, así como fomentar la enseñanza de habilidades creativas, incluidos los idiomas.

- Promoción de la cultura como elemento vital en las relaciones internacionales de la Unión: Mediante el diálogo político - y otras acciones - , la UE pretendía incorporar la dimensión cultural a sus distintas acciones y esfuerzos hacia el exterior, así como desarrollar nuevos proyectos propios del sector cultural.

A través de un marco de acciones que comprenden cuatro dimensiones (diálogo con el sector cultural, método de coordinación entre los Estados miembros y la UE, integración de la cultura en todas las políticas pertinentes, y el apoyo a los objetivos establecidos), la Comisión determinaba la importancia de fortalecer al sector cultural, así como integrar dicha dimensión a su política externa, atendiendo las necesidades y realidad del mundo globalizado.

Varios meses después, en noviembre del mismo año, el Consejo emitió su resolución, recibiendo la propuesta y “considerándola un paso importante hacia un mayor desarrollo de la cooperación en el ámbito cultural y un incremento de la coherencia y de la notoriedad de la actuación europea en este ámbito, al tiempo que se consolida el papel transversal de la cultura." (Consejo de la UE, 2007, pág. 1). De igual manera, establecía prioridad en la promoción del acceso a la cultura y movilidad de artistas, el desarrollo de datos para la evaluación, el rescate del potencial de las industrias creativas, así como la aplicación de la Convención firmada con la UNESCO en 2005.

\section{Programa Cultura (2007/2013) y el Año Europeo del Diálogo Intercultural (2008)}

Dentro de la Agenda Europea para la Cultura en un Mundo en vías de Globalización se mencionan dos proyectos a desarrollar en los siguientes años, el primero es el programa Cultura, con vigencia de 2007 a 2013 y el Año Europeo del Diálogo Intercultural que tendría lugar durante el 2008.

El primero tiene su antecedente en “Cultura 2000", vigente del año 2000 al 2006, posteriormente abierto para un nuevo periodo. Tenía por objetivo contribuir al desarrollo de un espacio cultural común a los 
ciudadanos de la Unión, con el fin de fortalecer la identidad europea. Mediante la financiación de proyectos de cooperación y el fomento de redes culturales, el programa apoyaba la movilización transnacional de artistas y profesionales del sector cultural, la promoción del diálogo intercultural y la circulación de bienes y servicios artísticos y culturales. Actualmente, Cultura, está vigente para el periodo 2014-2020 y opera en el marco del programa Europa Creativa. ${ }^{11}$

Por su parte, 2008 fue declarado el Año Europeo del Diálogo Intercultural, fundamentado en las ampliaciones de la Unión, la movilidad de los ciudadanos y la contribución de los países de la UE al desarrollo de las culturas. El proyecto tenía como principal objetivo aumentar el diálogo intercultural y la mejora en la coexistencia de sociedades diversas, al mismo tiempo que contribuía al fortalecimiento de la identidad europea. Por lo tanto, estaba dirigido únicamente a los Estados miembros, así como a los países candidatos.

Aunado a lo anterior, el proyecto sirvió como un piloto para otorgar un perfil visible a las buenas prácticas en los procesos de diálogo intercultural, con la finalidad de desarrollar estrategias sostenibles posteriores a este año.

Conclusiones del Consejo sobre la promoción de la diversidad cultural y el diálogo intercultural en las relaciones exteriores de la Unión Europea (2008)

Tras varias acciones de relación en la materia, como la Comunicación sobre una Agenda Europea de Cultura, la Convención firmada en 2005 con la UNESCO para la promoción y protección de la Diversidad de las Expresiones Culturales, entre otras; el Consejo de la UE y los representantes de los gobiernos de los Estados miembros emitieron sus conclusiones al respecto.

11. Europa Creativa es el programa marco de la Comisión Europea para apoyar a los sectores cultural y audiovisual, mediante los programa 'Cultura' y 'MEDIA'. Sus objetivos son el apoyo a ambos sectores para aprovechar oportunidades de la era digital y la globalización; lograr que realicen su potencial económico y contribuyan al crecimiento sostenible, empleo y cohesión social; así como brindarles acceso a nuevas oportunidades, mercados y públicos internacionales. Con la finalidad de contribuir a la cooperación al desarrollo en escala global, las convocatorias del programa también están abiertas para países no pertenecientes a la Unión (Europa Creativa, https:/ / ec.europa.eu/programmes/creative-europe/node_es). 
En éstas, el Consejo invitaba a los Estados miembros a trabajar por el reconocimiento de la cultura en sus políticas y programas, a fin de favorecer la cooperación en la materia con el resto del mundo y organizaciones competentes al tema, como la UNESCO y el Consejo de Europa. Sobre todo, invitaba a la "elaboración de una estrategia europea destinada a inscribir de forma coherente y sistemática a la cultura en las relaciones exteriores de la Unión y contribuir a que las acciones de la Unión sean complementarias con las de sus Estados miembros, establecimiento de estrategias específicas con las regiones y los países terceros, a fin de precisar los objetivos y medios de acción en materia de relaciones culturales" (Consejo de la Unión Europea, 2008, pág. 2).

Tras varios esfuerzos, tanto al interior de las instituciones de la UE, así como al exterior por parte de otros actores, el Consejo de la Unión por fin emitía y solicitaba avances en el camino hacia una estrategia cultural más unificada y acorde al nivel supranacional que representa.

Informe sobre las Dimensiones Culturales de las Acciones Exteriores de la Ue (2011)

En 2011, por iniciativa de la eurodiputada neerlandesa, Marietje Schaake, se presentó a la Comisión de Cultura y Educación del Parlamento Europeo, un informe sobre las Dimensiones Culturales de las Acciones Exteriores de la UE. Subrayaba la importancia y necesidad de reconocer el valor de la cultura en la política exterior europea, además llamaba a la urgencia de la cooperación en temas culturales (Parlamento Europeo, 2011).

En cuanto a los programas de la Unión, en el noveno punto del Informe: "Expresa su preocupación por la fragmentación de la política y los proyectos culturales de la UE, que obstaculiza el uso estratégico y eficaz de los recursos culturales y el desarrollo de una estrategia común visible de la UE en los aspectos culturales de las relaciones exteriores de la UE" (Parlamento Europeo, 2011). Si bien los Estados miembros de la Unión son fuertes en sus estrategias y políticas culturales a nivel nacional, al interior de las instituciones se percibía una falta de consolidación de una estrategia supranacional que, haciendo alusión a su lema "Unida en la Diversidad", posicionara a la UE en la esfera internacional. 
Al respecto, en el punto vigésimo segundo: “[se] Subraya la importancia de la diplomacia y la cooperación culturales en la promoción en todo el mundo de los intereses y de los valores de la UE y los Estados miembros que conforman la cultura europea; destaca la necesidad de que la UE actúe como protagonista con una perspectiva y una responsabilidad a escala mundial" (Parlamento Europeo, 2011).

Posteriormente, aboga por que las acciones de la Unión apunten hacia la promoción de la paz, y los derechos humanos, así como el desarrollo económico y el comercio internacional. Recomendaciones que serán tomadas en cuenta en la Acción Preparatoria, proyecto solicitado por el Parlamento para dar seguimiento a la propuesta cada vez más latente de una política cultural de la Unión Europea.

Como respuesta al informe, el Parlamento también apuntó hacia la necesidad de consolidar una política común en el tema cultural, previniendo la fragmentación de los esfuerzos individuales. En 2012, designa a la Comisión Europea y a un consorcio de expertos para la Acción Preparatoria sobre la Cultura en las Relaciones Exteriores de la UE (Unión Europea, 2014).

\section{Acción Preparatoria sobre 'La Cultura en las Relaciones Exteriores de la UE' $(2011 / 2014)$}

En mayo de 2011, la Comisión Europea, a petición del Parlamento, solicitó el proyecto de Acción Preparatoria sobre "La Cultura en las Relaciones Exteriores de la UE". La investigación, publicada en 2014 estuvo a cargo de un equipo de expertos liderado por el doctor Yudhishithir Raj Isar y el Instituto Goethe, y tenía por objetivo analizar la situación y el papel que jugaba la cultura - hasta el momento - en las relaciones exteriores de la Unión.

La investigación comprendió tanto a los 28 Estados miembros de la Unión, como a los 16 Estados beneficiarios de la Política de Vecindad y Asociación de la $U^{12}$ y a 10 países considerados aliados estratégicos ${ }^{13}$ debido a su relación con la Unión. El documento se estructura

12. Países de vecindad y asociación: Argelia, Armenia, Azerbaiyán, Bielorrusia, Egipto, Georgia, Israel, Jordania, Líbano, Libia, Marruecos, Moldavia, Palestina, Siria, Túnez y Ucrania.

13. Aliados estratégicos: Brasil, Canadá, China, Corea del Sur, Estados Unidos, India, Japón, México, Rusia y Sudáfrica. 
en cuatro secciones: un mapeo de los Estados miembros y sus acciones en el sector cultural, comprendiendo actores principales, modelos de trabajo, representación en el exterior, objetivos, herramientas de intervención y prioridades geográficas; un análisis de los Estados vecinos y aliados estratégicos; lecciones aprendidas gracias a las experiencias tanto a nivel nacional como supranacional respecto al papel de la cultura en la política exterior; y finalmente algunas recomendaciones para el futuro.

Es de relevancia mencionar que el documento incluye también una justificación teórica en torno a su acercamiento al campo a través del concepto de cultura en las relaciones exteriores, mucho más general que el término diplomacia cultural que es ampliamente utilizado en el escenario internacional.

De igual manera, la Acción Preparatoria se sustenta y trabaja a lo largo del documento en tres procesos clave, cuya presencia ha sido notoria y cada vez más evidente en los distintos esfuerzos previos hasta este momento en la historia de una política cultural de la Unión. El diálogo intercultural, el mutuo entendimiento, así como el empoderamiento y la prosperidad a través de la cultura son los elementos que la UE ha definido claves para justificar e integrar la cultura en su política exterior, pero que además ha totalizado en la noción de una ciudadanía cultural global (Unión Europea, 2014).

Al respecto del concepto de ciudadanía cultural global, se menciona en el reporte:

The notion of global cultural citizenship describes a process that meaningfully locates rights and responsibilities at the world scale, in an era when the exclusive link between citizenship and the single nation-state has been greatly weakened. It sees such rights and responsibilities as a horizon to be attained by humanity as a whole. Above all, it seeks the development of a global civil society and public sphere that is able to constructively 'negotiate difference' and foster a spirit of trans-national solidarity. As a goal to be pursued on the world stage 
cultural citizenship represents the needs and interests of both Europe and its partners ${ }^{14}$ (Unión Europea, 2014, pág. 22).

La investigación realizada por el consorcio de expertos se resume en 8 puntos clave (Unión Europea, 2014, pág. 13):

- A través de sus relaciones culturales, Europa tiene una enorme oportunidad para aumentar su influencia y atracción en el mundo.

- Tanto al interior de Europa, como en el mundo, existe una gran demanda de una estrategia europea de relaciones culturales.

- Dicha estrategia debe ser coherente, considerando que no se refiere únicamente a presentar la cultura europea al mundo sino que además debe integrarse en un proceso colaborador con otros actores, escuchándolos, compartiendo y diseñando nuevos métodos creativos.

- La estrategia de la UE debe considerar y ser congruente con los intereses y prácticas de las nuevas generaciones, quienes, gracias a los avances tecnológicos han sobrepasado las barreras de la comunicación transnacional inmediata.

- Dicha estrategia debe ser transversal e integral, tomando en cuenta a los distintos actores, tanto las instituciones de la UE, como los institutos de promoción cultural de los Estados miembros y la sociedad civil, en una estrategia que persiga el ideal de una ciudadanía cultural global.

- Se requiere el compromiso de todos los participantes. La UE deberá financiar el proyecto y éste deberá ser diseñado por expertos en el sector cultural.

- Es necesario desarrollar una serie de prototipos y proyectos piloto que permitan evaluar y corregir la estrategia previo a su lanzamiento.

14. Traducción personal: "La noción de una ciudadanía cultural global describe un proceso que posiciona de manera significativa los derechos y responsabilidades en una escala global, en una era en donde la relación exclusiva entre la ciudadanía y el Estado-nación se ha debilitado. Visualiza dichos derechos y responsabilidades en un horizonte que debe ser alcanzado por la humanidad en su conjunto. Sobre todo, busca el desarrollo de una sociedad civil global y una esfera pública que es capaz de 'negociar constructivamente las diferencias' y fomentar un espíritu de solidaridad transnacional. Como meta para alcanzarse en la escena mundial, la ciudadanía cultural representa las necesidades e intereses tanto de Europa como de sus aliados". 
- La estrategia debe establecer objetivos claros y realistas, así como concebirse como un proyecto cuyos resultados serán percibidos en el largo plazo.

Los resultados de la investigación fueron discutidos a nivel de la Unión Europea en una conferencia que se llevó a cabo en Bruselas el 7 y 8 de abril de 2014 .

Hacia una estrategia de relaciones culturales internacionales de la Unión Europea

Si bien hasta entonces no existía una declaración que integrara formalmente a la cultura como un pilar dentro de la política exterior de la UE, ésta siempre ha sido una parte fundamental en su proceso de integración. En sus diferencias, la Unión ha encontrado sus mayores fortalezas, logrando posicionarse como un actor importante en el sistema internacional. Por lo anterior, "promover la diversidad a través de las relaciones culturales internacionales reviste gran importancia para el papel de la UE como actor global" (Comisión Europea, 2016, pág. 2).

Las acciones anteriores lograron establecer el camino de la Unión hacia una estrategia para las relaciones culturales internacionales. En junio de 2016, la Comisión Europea, a través de Federica Mogherini, Alta Representante de la Unión para Asuntos Exteriores y Política de Seguridad, envió al Parlamento Europeo y al Consejo, la Comunicación Conjunta que lleva dicho nombre. En ella, establece la importancia y el compromiso de integrar formalmente una estrategia cultural dentro del marco de la Unión.

El documento, fiel a la Agenda Europea para la Cultura y la Acción Preparatoria sobre "La Cultura en las Relaciones Exteriores de la UE", reconoce nuevamente la necesidad del diálogo intercultural como herramienta para la paz, el papel de las industrias culturales y creativas en el desarrollo económico y -como su nombre lo indica - la importancia de la cultura en las relaciones internacionales de la Unión. Específicamente, dicho documento:

[...] se propone una estrategia de la UE para las relaciones culturales internacionales que se centra en impulsar la cooperación cultural con los países socios en torno a tres ejes principales: el apoyo de la cultura como motor del desarrollo económico y social sostenible; el fomento de la cultura y del diálogo intercultural 
para las relaciones intercomunitarias pacíficas; el refuerzo de la cooperación en el patrimonio cultural. Con el fin de alcanzar estos objetivos, las relaciones culturales internacionales de la UE contribuirán a hacer de la UE un actor más potente en el escenario mundial (Comisión Europea, 2016, pág. 3).

Se comprende, entonces, que la Comisión adopta uno de los tres ejes mencionados anteriormente (la cultura en las relaciones internacionales) e integra uno más a las prioridades, que si bien se había mencionado en comunicaciones pasadas no ocupaba un lugar protagonista: la protección del patrimonio cultural.

La comunicación establece, además, cinco principios que guiarán la acción de la Unión en el ámbito de las relaciones culturales internacionales (Comisión Europea: 2016, pág. 4):

- Promoción de la diversidad cultural y el respeto de los derechos humanos.

- Fomento del respeto mutuo y el diálogo intercultural.

- Garantía del respeto a la complementariedad y subsidiariedad.

- Impulso de un enfoque transversal de la cultura.

- Promoción de la cultura a través de los marcos de cooperación existentes.

Sobre lo anterior se establece que la UE respeta la soberanía de sus Estados miembros en cuanto a sus políticas culturales, no obstante se hace presente para complementar y coordinar los esfuerzos en el nivel supranacional. Además, se entiende por cultura no únicamente a las bellas artes, sino que se abre a un sinfín de actividades, así como a la comprensión del concepto desde la parte antropológica del mismo la cual determina que la cultura es además el conjunto de tradiciones, visiones y creencias de los pueblos.

Respecto al último punto, cabe mencionar que la UE en su marco de cooperación ha establecido una línea de programas temáticos ${ }^{15} \mathrm{y}$

15. Los programas temáticos en el marco de cooperación de la UE comprenden el Instrumento de Colaboración (IC), el Instrumento Europeo para la Democracia y los Derechos Humanos (IEDDH), el Instrumento de Cooperación al Desarrollo (ICD), el Instrumento en pro de la estabilidad y la paz (IEP) y el Programa Europa Creativa. 
geográficos, ${ }^{16}$ a través de los cuales se propone "garantizar la coherencia de las políticas y evitar su duplicación, la manera más eficaz de promover la cultura en las relaciones exteriores de la UE consiste en utilizar los marcos de cooperación y los instrumentos de financiación existentes." (Comisión Europea, 2016, pág. 5).

Como marco de acción para respaldar, impulsar y alcanzar los objetivos propuestos en la estrategia, se proponen tres líneas de trabajo: Apoyo a la cultura como motor del desarrollo económico y social sostenible: Mediante el diseño de políticas culturales, el fortalecimiento de las industrias creativas (con especial énfasis en las pymes) y la ayuda en la materia, a los países socios.

- Fomento de la cultura y el diálogo intercultural para las relaciones intercomunitarias pacíficas: A través del fomento de la cooperación cultural, tanto entre Estados como entre los distintos actores del sector cultural, así como la promoción de la paz mediante el diálogo. - Refuerzo de la cooperación en materia de patrimonio cultural: A través de la investigación y el intercambio de experiencias entre los actores, se pretende rehabilitar y promover el patrimonio cultural tanto de Europa como de terceros países.

La evidencia en el recorrido histórico de Europa demuestra que la Unión Europea no parte desde cero en sus esfuerzos de integración de una estrategia de relaciones culturales internacionales; por el contrario, los países europeos se distinguen por ser pioneros en el ámbito de la cultura en política exterior. Al respecto, la Unión defiende el principio de complementariedad e invita a la suma de los esfuerzos existentes, como los distintos institutos culturales de sus Estados miembros, así como organismos y fundaciones independientes a la UE. Lo anterior ha impulsado que dentro de la estrategia se comprendan dos enfoques: el refuerzo de la cooperación de la UE y los intercambios culturales de estudiantes, investigadores y alumnos (Comisión Europea, 2016).

16. El Marco Geográfico de Cooperación de la UE incluye los Países ACP (África, Caribe y Pacífico), la Política de Pre-Adhesión (IPA), Política Europea de Vecindad y Asia y América Latina dentro del Instrumento de Cooperación al Desarrollo (ICD). 
Del primer enfoque se desprende la colaboración con otros organismos como EUNIC y la Fundación Anna Lindh, ${ }^{17}$ así como un proyecto de especial interés, la plataforma de Diplomacia Cultural. En cuanto al segundo enfoque, durante el periodo 2014/ 2020, los intercambios académicos se fomentan a través de las Acciones Marie SklodowskaCurie (AMSC) ${ }^{18}$ y el programa Erasmus $+{ }^{19}$ ambos comprendidos en la Dirección General de Educación y Cultura.

\section{Plataforma de Diplomacia Cultural}

Lanzada en marzo de 2016, la plataforma tiene la finalidad de apoyar a las instituciones de la Unión Europea en la implementación de la estrategia de relaciones culturales internacionales, especialmente "to gather all the actors - governments, regions, cities, cultural institutes, civil society organizations, artists, scientists, performers, individuals and many more - of the European external cultural relations, and engage them on a continuous basis, receive feedback, policy advice and support." ${ }^{20}$ (Mogherini, Cultural Diplomacy Platform, sin fecha). pales:

La plataforma define su actuar con base en tres objetivos princi-

Apoyar y fomentar el desarrollo de las políticas, actividades, metodologías y herramientas de la diplomacia cultural, dentro del marco general de la diplomacia pública europea.

17. Creada en 2005, la Fundación Anna Lindh es una institución intergubernamental dedicada a la promoción del diálogo intercultural en la región Euro-Mediterránea, con la finalidad de fomentar el respeto y mutuo entendimiento entre las culturas, así como la confianza entre las naciones y sus ciudadanos (Anna Lindh Foundation, Mandate and Founders [http:/ / www.annalindhfoundation.org/mandate-and-founders]).

18. Dentro del marco Horizonte 2020, las Acciones Marie Sklodowska-Curie (AMSC) tienen la finalidad de fomentar el desarrollo profesional y la formación de investigadores de varias disciplinas científicas, tanto de los ciudadanos de la UE, como aquellos que no pertenecen (Fundación EULAC, 2017).

19. Erasmus+ es el programa de la UE para la movilidad de estudiantes de distintos niveles académicos (europeos y no europeos) y distintos apoyos a las Instituciones de Educación Superior europeas (Fundación EULAC, 2017).

20. Traducción personal: "para reunir a los distintos actores - gobiernos, regiones, ciudades, instituciones culturales, organizaciones de la sociedad civil, artistas, científicos, intérpretes, individuos y muchos más - de las relaciones culturales externas de Europa, e involucrarlos en el proceso de manera continua, recibir retroalimentación, asesoramiento y apoyo en políticas culturales. 
- Fomentar el diálogo político y asesorar en temas de diplomacia cultural.

- Fortalecer las comunidades y redes de diplomacia cultural.

A través del Consorcio ${ }^{21}$ responsable de la implementación de la plataforma, ésta toma en cuenta la importancia de involucrarse con terceros países y ofrece asesoramiento a las instituciones de la UE en el establecimiento y desarrollo de las relaciones culturales externas. Actualmente, sus esfuerzos se centran en los 10 socios estratégicos definidos en la Acción Preparatoria (Brasil, Canadá, China, Corea del Sur, Estados Unidos, India, Japón, México, Rusia y Sudáfrica).

\section{Conclusiones}

En las últimas décadas, la cultura se ha posicionado como uno de los pilares fundamentales en el panorama de las relaciones internacionales. Esto, debido a diversos factores como el uso de las diferencias culturales para la movilización y politización de los conflictos, su potencial dentro del desarrollo sostenible (de gran importancia para la Unión debido a su compromiso con los Objetivos del Milenio), así como el surgimiento de nuevos actores y tecnologías que permiten interacciones transnacionales cada vez más rápidas y frecuentes.

Si bien tanto la cultura como la diversidad han sido eje central en el proceso de integración de la Unión Europea, hasta el día de hoy no se encontraban formalmente integradas en su política exterior. No obstante, la creciente demanda, tanto al interior como al exterior del continente, así como el avanzado nivel de integración, han encaminado a la Unión al que parece ser el siguiente paso natural: la alineación de los distintos esfuerzos nacionales y de actores no-estatales, en una estrategia común de relaciones culturales internacionales. Cabe mencionar que la preferencia de la UE por dicho término sobre el de diplomacia cul-

21. Debido a que la plataforma se definió bajo los términos y en el marco de la "Acción Preparatoria 'Cultura en las Relaciones Exteriores de la UE', el consorcio responsable es el mismo. Está integrado por el Instituto Goethe, el British Council, EUNIC, la Fundación Europea de la Cultura, el Instituto Francés y el Palacio de Bellas Artes/BOZAR. 
tural, le permite una mayor actuación en el campo a través de distintas herramientas, participación de actores y establecimiento de objetivos.

A lo largo del recorrido de distintas acciones en materia cultural, se observa una continuidad en los fines establecidos en la Unión. Desde 2007, con la Agenda Europea para la Cultura, se establece la importancia de la dimensión cultural como elemento esencial en las relaciones internacionales de la UE, aunado al diálogo intercultural para la promoción de la paz y los derechos humanos, así como el fortalecimiento de las industrias creativas y culturales debido a su influencia en el desarrollo sustentable mediante la creación de empleos y la cohesión social.

Los anteriores estarán presentes en todo el proceso hacia una estrategia de relaciones culturales internacionales. Si bien mencionados de manera distinta, es posible encontrar las coincidencias. Ya en 2006, la Fundación Europea de Cultura hablaba de visibilidad, seguridad y desarrollo económico, a través del diálogo intercultural y la promoción de los valores europeos al exterior. Posteriormente, la eurodiputada neerlandesa, Marietje Schaake, en su informe sobre las Dimensiones Culturales en las Acciones Exteriores de la UE, en 2011, abogaría por la promoción de la paz y los derechos humanos, el desarrollo económico y el comercio internacional mediante las acciones culturales de la Unión.

El mismo año, la Acción Preparatoria sobre "La Cultura en las Relaciones Exteriores de la UE" mencionaba el diálogo intercultural, el mutuo entendimiento y el empoderamiento y la prosperidad a través de la cultura, como elementos clave en su integración a la acción exterior de la Unión. Posteriormente el 8 de junio de 2016, la Comisión adoptaría los mismos principios, adjuntando la importancia de la cooperación en materia del patrimonio cultural.

Ante la evidencia de la coherencia en los fines y usos de la cultura en la acción exterior, lo anterior sugiere que dicha estrategia ha estado presente desde muchos años atrás, pero se encontraba dividida en los distintos esfuerzos nacionales. No obstante, el documento "Hacia una estrategia de la UE para las relaciones culturales internacionales", es evidencia de pasos concretos hacia una política común en el tema.

Lo anterior permitirá posicionar a la Unión como un agente global, consintiéndole la promoción de sus valores en el exterior con mucha más presencia, así como el fortalecimiento de su relación con terceros países mediante la cooperación cultural, al mismo tiempo que contribuye a la noción de una ciudadanía cultural global. Europa y la Unión, 
son mucho más que un espacio geográfico, son una idea, una unidad y un proceso vivo.

\section{Referencias}

Anna Lindh Foundation (sin fecha). Consultado el día 15 de mayo de 2017 en [http://www.annalindh foundation.org/]

Bound, K.; Briggs, R.; Holden, J.; Jones, S. (2007). Culture is a central component of international relations, It's time to unlock its full potential... Londres: DEMOS.

Comisión Europea (2007). Comunicación de la Comisión al Parlamento Europeo, al Consejo, al Comité Económico y Social Europeo y al Comité de las Regiones - Comunicación sobre una Agenda Europea para la Cultura en un Mundo en vías de Globalización \{SEC (2007) 570\}. Bélgica. COM (2007) 242 final. Consultado el 9 de mayo de 2017 en [http:/ / eur-lex.europa.eu/ legal-content/ES/TXT/PDF/?uri=CELEX:52007DC0242\&from=ES]

Comisión Europea; Alta Representante de la Unión Europea para Asuntos Exteriores y Política de Seguridad (2016). Comunicación Conjunta al Parlamento Europeo y al Consejo - Hacia una estrategia de la UE para las relaciones culturales internacionales. Bélgica. JOIN (2016) 29 final. Consultado el 9 de mayo de 2017 en [http:/ / eur-lex.europa.eu/legal-content/ ES/TXT/PDF/?uri=CELEX:52016JC0029\&from=ES]

Comisión Europea (2010). Año Europeo del Diálogo Intercultural (2008). Consultado el día 11 de mayo de 2017 en [http://eur-lex.europa.eu/ legal-content/ES/TXT/?uri=uriserv\%3Al29017]

Comisión Europea (2014). Preparatory Action 'Culture in EU External Relations'. Engaging the World: Towards Global Cultural Citizenship. Consultado el 11 de mayo de 2017 en [http://ec.europa.eu/assets/eac/culture/library/publications/global-cultural-citizenship_en.pdf]

Comisión Europea (2017). Europa Creativa. Consultado el 16 de mayo de 2017 en [https:/ / ec.europa.eu/programmes/creative-europe/node_es]

Comisión Europea (2017). Marco estratégico: Agenda Europea para la Cultura. Consultado el día 14 de mayo de 2017 en [https://ec.europa.eu/ culture/policy/strategic-framework_es]

Comisión Europea (2017). Preparatory Action for Culture in External Relations. Consultado el día 14 de mayo de 2017 en [https://ec.europa.eu/ culture/initiatives/external-relations_es]

Consejo de la Unión Europea (2007). Resolución del Consejo de 16 de noviembre de 2007 relativa a una Agenda Europea para la Cultura (2007/C 287/01). Diario Oficial de la Unión Europea. Consultado el día 11 de mayo 
de 2017 en [http://eur-lex.europa.eu/legal-content/ES/TXT/PDF/?uri $=$ CELEX:32007G1129(01)\&from $=$ ES]

Consejo de la Unión Europea (2008). Conclusiones del Consejo y de los representantes de los Gobiernos de los Estados miembros, reunidos en el seno del Consejo, sobre la promoción de la diversidad cultural y el diálogo intercultural en las relaciones exteriores de la Unión Europea y de sus Estados miembros (2008/C 320/ 04). Diario Oficial de la Unión Europea. Consultado el día 11 de mayo de 2017 en [http:/ / eur-lex.europa.eu/ legal-content/ES/TXT/PDF/?uri=CELEX:42008X1221(02)\&from=ES]

Consejo de Europa (2011). Living together, Combining diversity and freedom in 21st Century Europe. Report of the Group of Eminent Persons of the Council of Europe. Consultado el 17 de octubre de 2016 en [https:/ / www.coe.int/t/dg4/highereducation/2011/KYIV\%20WEBSITE/Report $\% 20$ on $\% 20$ diversity.pdf]

Consejo de Europa (2017). Culture and Cultural Heritage. European Cultural Convention (Paris, 1954). Consultado el día 10 de mayo de 2017 en [http://www.coe.int/en/web/culture-and-heritage/european-cultural-convention]

Consejo de Europa (2017). Intercultural cities programme. Consultado el día 11 de mayo de 2017 en [http://www.coe.int/en/web/interculturalcities/]

Consejo de Europa/ERI Carts "Compendium of Cultural Policies and Trends in Europe". (2016). Council of Europe. Consultado el día 12 de mayo de 2017 en [http:/ / www.culturalpolicies.net/web/coe.php]

Cummings, M.C. (2003). Cultural Diplomacy and the United States Government: A Survey. Washington DC: Americans for the Arts. pp.15

EUNIC (2015). EUNIC Strategic Framework. Consultado el día 10 de mayo de 2017 en [https://www.eunic-online.eu/sites/default/files/imce/ u1264/strategic_framework_adopte d.pdf]

European Union, Cultural Diplomacy Platform. (2016). Consultado el día 15 de mayo de 2017 en [http:/ / www.cultureinexternalrelations.eu/]

Fontaine, P. (2014). Doce lecciones sobre Europa, comprender las políticas de la Unión Europea. Comisión Europea, Acción Exterior de la UE 21, pp.48.

Fundación EU-LAC (2017). Vademécum sobre Programas de Cooperación de la UE con ALC. Alemania. pp.89

Gorzegno, O. (27/04/2017). La Integración Europea. Un largo camino accidentado. Conferencia presentada en la Universidad de Guadalajara para la Maestría en Relaciones Económicas Internacionales y Cooperación.

Isar, Y.R. (02/05/2013). "Culture in External Relations": The EU in Cultural Diplomacy. En Organización para la Seguridad y la Cooperación en Europa, Trabajo presentado en "OSCE Talks seminar 'Cultural diplomacy in a 
global digital age'": Estambul, Turquía. (14p.). Recuperado el 11 de mayo de 2017 en [http:/ / www.osce.org/secretariat/103748?download=true]

Martín de la Guardia, R.; Pérez, G. (2001). “Europa, Mosaico de Tierras y Pueblos: Unidad en la Diversidad" en Historia de la Integración Europea (pp. 9-13). España: Ariel.

Nye, J.S. (2016). El poder suave. La clave del éxito en la política internacional. Traducción, Rossana Reyes. México: Universidad Iberoamericana.

Parlamento Europeo (2011). Informe sobre las dimensiones culturales de las acciones exteriores de la UE. Ponente: Marietje Schaake ante la Comisión de Cultura y Educación. Consultado el día 12 de mayo de 2017 en [http:/ / www.europarl.europa.eu/sides/getDoc.do?pubRef=-//EP// TEXT+REPORT+A7-2011-0112+0+DOC+XML+V0//ES\#title1]

Pantea, D.; Stoica, A. (2014). The role of cultural diplomacy in contemporary crises and conflicto reconcilition. Studia Universitatis Babes-Bolyai Studia Europapea, pp. 219-230.

Paschalidis, G. (2009). Exporting National Culture: Histories of Cultural Institutes Abroad. International Journal of Cultural Policy, Vol. 15 (No. 03). pp. 275-289.

Programa de Naciones Unidas para el Desarrollo (PNUD). (2005). ‘La Libertad Cultural en el Mundo Diverso de Hoy', en Informe sobre Desarrollo Humano 2004. La Libertad Cultural en el Mundo Diverso de Hoy. Ed: Mundi-Prensa. PNUD. pp. 1-12

Roulet, S. Etimología de Europa en Diccionario Etimológico español en línea. Consultado el día 29 de abril de 2017 en [http:/ / etimologías.dechile. net/?Europa]

Saddiki, S. (2009). El papel de la diplomacia cultural en las relaciones internacionales. Revista CIDOB d'Afers Internationals. Vol. 88. pp. 107-118

UNESCO (2005). Convención sobre la protección y promoción de la Diversidad de las Expresiones Culturales. París. 19p.

Unión Europea. (26/12/2012). Versiones Consolidadas del Tratado de la Unión Europea y del Tratado de Funcionamiento de la Unión Europea (2012/C 326/01). Diario Oficial de la Unión Europea. Recuperado el 12 de mayo de 2017 en [http://eur-lex.europa.eu/legal-content/ES/TXT/ PDF/?uri=CELEX:12012E/TXT\&from=ES] 